\title{
Inner rings in disc galaxies: dead or alive ${ }^{\star, \star \star}$
}

\author{
S. Comerón ${ }^{1,2}$ \\ ${ }^{1}$ University of Oulu, Astronomy Division, Department of Physics, PO Box 3000, 90014 Oulu, Finland \\ e-mail: seb.comeron@gmail.com \\ ${ }^{2}$ Finnish Centre of Astronomy with ESO (FINCA), University of Turku, Väisäläntie 20, 21500 Piikkiö, Finland \\ Received 28 May 2013 / Accepted 17 June 2013
}

\section{ABSTRACT}

\begin{abstract}
In this Letter, I distinguish "passive" inner rings as those with no current star formation as distinct from "active" inner rings that have undergone recent star formation. I built a sample of nearby galaxies with inner rings observed in the near- and mid-infrared from the NIRSOS and the $\mathrm{S}^{4} \mathrm{G}$ surveys. I used archival far-ultraviolet (FUV) and $\mathrm{H} \alpha$ imaging of 319 galaxies to diagnose whether their inner rings are passive or active. I found that passive rings are found only in early-type disc galaxies $(-3 \leq T \leq 2)$. In this range of stages, $21 \pm 3 \%$ and $28 \pm 5 \%$ of the rings are passive according to the FUV and $\mathrm{H} \alpha$ indicators, respectively. A ring that is passive according to the FUV is always passive according to $\mathrm{H} \alpha$, but the reverse is not always true. Ring-lenses form $30-40 \%$ of passive rings, which is four times more than the fraction of ring-lenses found in active rings in the stage range $-3 \leq T \leq 2$. This is consistent with both a resonance and a manifold origin for the rings because both models predict purely stellar rings to be wider than their star-forming counterparts. In the case of resonance rings, the widening may be at least partly due to the dissolution of rings. If most inner rings have a resonance origin, I estimate $200 \mathrm{Myr}$ to be a lower bound for their dissolution time-scale. This time-scale is of the order of one orbital period at the radius of inner rings.
\end{abstract}

Key words. galaxies: evolution - galaxies: kinematics and dynamics - galaxies: spiral - galaxies: statistics

\section{Introduction}

Gas in disc galaxies is redistributed by angular momentum transfer caused by nonaxisymmetries with a given pattern speed such as bars, ovals, and spiral arms. Some of the gas is collected in orbits near dynamical resonances under the influence of the torques caused by the nonaxisymmetries (for a recent review on barred galaxy dynamics see Athanassoula 2012a). Owing to star formation triggered by the high gas density and by gas travelling in intersecting orbits at each side of the resonance, rings and pseudorings are often formed there (Schwarz 1981, 1984). Historically, this picture has been used to explain resonance rings and pseudorings, but recently an alternative model, called the flux tube manifold theory or manifold theory, postulated that at least some of them are caused by gas and/or stars trapped in tubes of orbits that connect the Lagrangian points at the end of the galaxy bars (Romero-Gómez et al. 2006, 2007; Athanassoula et al. 2009b,a, 2010; Athanassoula 2012b). Another alternative ring formation mechanism is that developed by Kim et al. (2012) for nuclear rings (not studied here), where rings are formed due to the centrifugal barrier encountered by gas migrating to inner regions of the galaxy. In this Letter, the word rings is used to refer to both rings and pseudorings.

In the classical resonance theory and also in the manifold theory, inner rings in barred galaxies have a diameter slightly larger than the bar length. In the classic resonance theory they are associated to the ultraharmonic 4:1 resonance. In this Letter, broad features intermediate between inner lenses and inner

\footnotetext{
* Appendix $\mathrm{A}$ is available in electronic form at http: //www . aanda . org

$\star \star$ Table A.1 is also available at the CDS via anonymous ftp to cdsarc.u-strasbg. fr $(130.79 .128 .5)$ or via http://cdsarc.u-strasbg.fr/viz-bin/qcat?]/A+A/555/L4
}

rings, called ring-lenses, are accounted for alongside inner rings. Simulations by Rautiainen \& Salo (2000) showed that inner rings, although changing in shape and size, are long-lived, provided there is a gas inflow to feed them. In the manifold theory they are also long-lived as long as the galaxy potential does not evolve, or does so slowly (Athanassoula et al. 2010).

"Passive" inner rings are here those not found to host star formation. In the context of nuclear rings, passive rings have also been called fossil rings (Erwin et al. 2001). As opposed to passive features, "active" rings are those with indications of recent star formation. An example of a passive inner ring in the literature is that in NGC 7702 (Buta 1991). Here, I study inner rings identified in two infrared surveys of nearby galaxies, namely the Spitzer Survey of Stellar Structure in Galaxies $\left(S^{4} G\right.$; Sheth et al. 2010) and the Near-InfraRed atlas of S0-Sa galaxies (NIRS0S; Laurikainen et al. 2011). The $S^{4} G$ is a survey of 2352 nearby galaxies in 3.6 and $4.5 \mu \mathrm{m}$ using the Spitzer Space Telescope and NIRSOS is a $K_{\mathrm{s}}$-band ground-based survey of 206 earlytype galaxies. To investigate whether rings are passive or active, I used archival images in one band and one line that trace recent star formation, namely the far-ultraviolet (FUV) and $\mathrm{H} \alpha$.

This Letter is structured as follows. In Sect. 2, I present the sample, the data, and the image processing. Then, I present the results in Sect. 3 and discuss them in Sect. 4. The conclusions are summarized in Sect. 5.

\section{Data selection and processing}

I mainly based my sample of galaxies with inner rings on the classification of $\mathrm{S}^{4} \mathrm{G}$ galaxies made by Buta et al. (in prep.) and statistically studied in the Atlas of Resonance (pseudo)Rings As Known In the $S^{4} G$ (ARRAKIS; Comerón et al., in prep.). Since 
the $S^{4} \mathrm{G}$ sample is biased against galaxies with a small gas fraction, which are mostly elliptical and S0 galaxies, I also included NIRSOS galaxies with inner rings that matched the $S^{4} \mathrm{G}$ selection criteria, namely galactic latitude $|b|>30^{\circ}$, radial velocity $v_{\mathrm{r}}<4000 \mathrm{~km} \mathrm{~s}^{-1}$, angular diameter $D_{25}>1^{\prime}$, and integrated blue magnitude $m_{\mathrm{B}}<15.5 \mathrm{mag}$ (data obtained from HyperLeda; Paturel et al. 2003). $S^{4} G$ and NIRSOS data can be mixed safely because the detection of inner rings in the $S^{4} G$ matches that in NIRSOS very well (Sect. 5.10 in ARRAKIS). I also included NGC 2950, an S0 non- $S^{4} G$ galaxy appearing in the same frame as a genuine $S^{4} \mathrm{G}$ galaxy which also fulfils the selection criteria.

To avoid dust absorption, ring foreshortening, and poor angular resolution problems, I additionally constrained the sample by only selecting disc galaxies (Hubble stage $-3 \leq T \leq 9$ ) with an ellipticity lower than $\epsilon_{\mathrm{d}}=0.5$ according to the data of the Pipeline 4 of $S^{4} \mathrm{G}$ (Salo et al., in prep.) and with inner rings with a radius larger than $10^{\prime \prime}$ according to ARRAKIS or NIRSOS. The total number of galaxies fulfilling these conditions is 357 .

Two indicators were used to search for recent star formation: the far-ultraviolet continuum and $\mathrm{H} \alpha$-line emission.

The ultraviolet continuum traces star formation that has occurred in the past $100 \mathrm{Myr}$ (Kennicutt 1998). To study inner rings in that wavelength, I downloaded the deepest available FUV-band image in the GALEX GR6/7 Data Release ${ }^{1}$ for each galaxy. Such images were available for 319 out of the 357 galaxies initially included in the sample. These 319 galaxies are the final sample I worked with. For 160 galaxies, the FUV images belong to the GALEX All-Sky Imaging Survey (AIS), which consists of $\sim 100 \mathrm{~s}$ exposures and can detect point sources down to $\mu_{\mathrm{AB}} \sim 20 \mathrm{mag} \operatorname{arcsec}^{-2}$. The other galaxies were imaged in deeper GALEX surveys and were in general exposed for $1000 \mathrm{~s}$ or more.

The $\mathrm{H} \alpha$ emission traces star formation that has occurred in the past $20 \mathrm{Myr}$ (Kennicutt 1998). H $\alpha$ continuum-subtracted images used here come from three sources:

- Images processed for the Atlas of Images of NUclear Rings (AINUR; Comerón et al. 2010). The images in AINUR come mostly from the Hubble Space Telescope (HST) Archive ${ }^{2}$.

- HST images not processed for AINUR. In these cases, $\mathrm{H} \alpha$ narrow-band images and red continuum images were downloaded from the Hubble Legacy Archive ${ }^{3}$ (HLA) and were used to produce a continuum-subtracted image using the technique described in Knapen et al. (2004, 2006).

- Continuum-subtracted images in the NASA/IPAC Extragalactic Database (NED) ${ }^{4}$.

$\mathrm{H} \alpha$ images were available for 139 out of the 319 sample galaxies.

For each sample galaxy I verified in the FUV and $\mathrm{H} \alpha$ continuum-subtracted images whether the rings detected in $S^{4} \mathrm{G}$ or NIRSOS images were visible. A detection was considered to be positive if at least a segment of the ring was seen. In some doubtful cases with shallow AIS FUV images, this was only possible after smoothing the image with a Gaussian kernel with a 3-pixel (4.5") radius. Positive detections are labelled as "A" for "active" and negative detections are labelled as "P" for "passive" in Table A.1. In doubtful cases, a "?" sign is added to the detection status. From now on, I refer to positive and negative

\footnotetext{
1 http://galex.stsci.edu/GR6/

2 http://archive.stsci.edu/hst/search.php

3 http://hla.stsci.edu/hlaview.html

4 http://ned.ipac.caltech.edu/
}

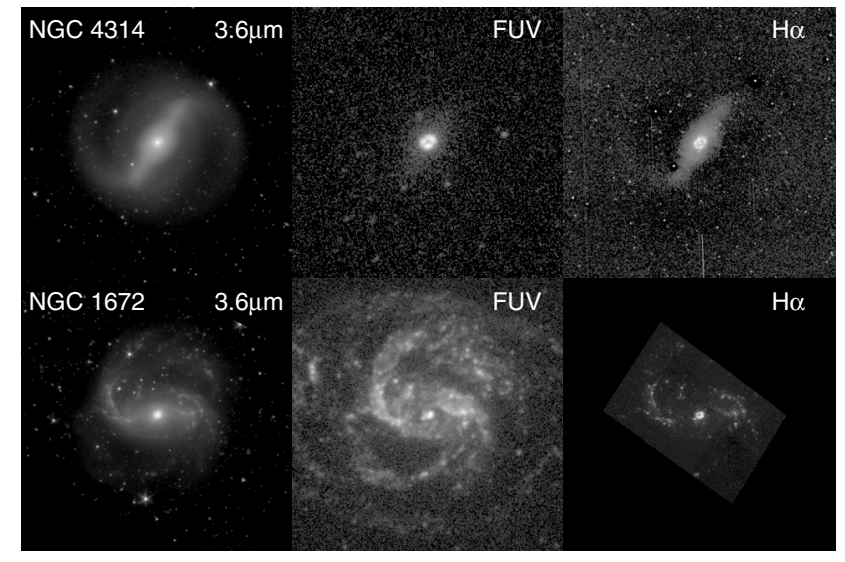

Fig. 1. NGC 4314 (top row) is an $\left(\mathrm{R}_{1}^{\prime}\right) \mathrm{SB}(\mathrm{rl}, \mathrm{nr})$ a galaxy (Buta et al., in prep.) with a passive inner ring-lens. NGC 1672 (bottom row) is an $\left(\mathrm{R}^{\prime}\right) \mathrm{SA} \underline{\mathrm{B}}(\mathrm{rs}, \mathrm{nr}) \mathrm{b}$ galaxy (Buta et al., in prep.) whose inner pseudoring is still forming stars. In the first case the inner feature does not emit in the FUV and $\mathrm{H} \alpha$. There is a significant amount of emission in these bands for the active inner feature. The prominent ring feature in NGC 4314 is a nuclear ring. The $3.6 \mu \mathrm{m}$ images are from the $\mathrm{S}^{4} \mathrm{G}$, the FUV images come from GALEX's NGS survey, and the $\mathrm{H} \alpha$ images come from Knapen et al. (2004) and the HLA.

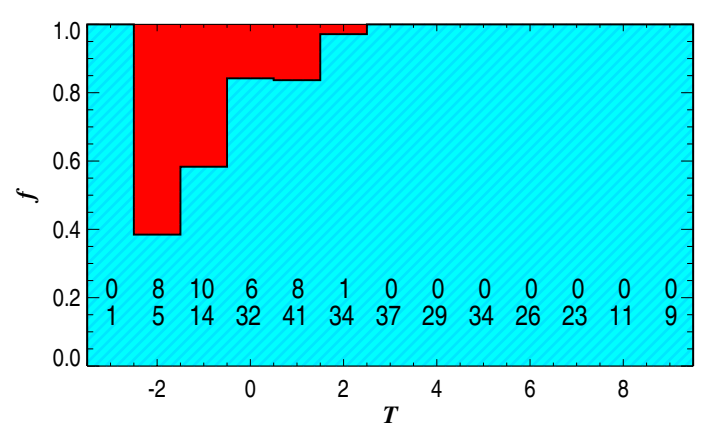

Fig. 2. Fraction of active and passive inner rings (blue hatched and red plain surfaces, respectively) according to the FUV indicator as a function of the galaxy stage for galaxies in the sample with available GALEX imaging. The bottom row of numbers indicate the number of active rings for a given stage and the top row indicates the number of those that are passive.

detections as active and passive rings, respectively. This definition means that some rings may be passive in one of the studied indicators but active in the other. Examples of galaxies with passive and active inner rings are shown in Fig. 1. A total of 329 inner rings in the 319 sample galaxies were analysed.

\section{Results}

Out of 329 inner rings, 33 were found not to have FUV emission (Fig. 2). All of them are in the stage range $-3 \leq T \leq 2$. The fraction of passive inner rings in this range of stages according to the FUV star formation indicator is $21 \pm 3 \%$ with the error bar calculated using binomial statistics. Rings hosted in early-type galaxies are more likely to be passive than those in later types (Table 1). I verified whether non-detections may be partly caused by the use of shallow AIS images by recalculating the statistics with deep GALEX images only (Table 1) and found that the results based on those two samples are compatible within the error bars, which indicates that rings with recent star formation can be detected even in relatively shallow FUV images. 
Table 1. Fraction of passive rings according to different star formation indicators and for different Hubble stage ranges.

\begin{tabular}{lcccc}
\hline \hline Indicator & All types & S0 & Early sp. & Late sp. \\
& $-3 \leq T \leq 9$ & $-3 \leq T \leq 0$ & $1 \leq T \leq 2$ & $3 \leq T \leq 9$ \\
\hline FUV & $10 \pm 2 \%$ & $32 \pm 5 \%$ & $11 \pm 3 \%$ & $0 \%$ \\
Deep FUV & $13 \pm 3 \%$ & $36 \pm 8 \%$ & $15 \pm 5 \%$ & $0 \%$ \\
FUV $(\mathrm{H} \alpha)$ & $8 \pm 2 \%$ & $16 \pm 7 \%$ & $16 \pm 6 \%$ & $0 \%$ \\
$\mathrm{H} \alpha$ & $13 \pm 3 \%$ & $48 \pm 10 \%$ & $16 \pm 6 \%$ & $0 \%$ \\
\hline
\end{tabular}

Notes. Deep FUV stands for GALEX FUV imaging excluding images from the AIS survey. FUV $(\mathrm{H} \alpha)$ stands for the FUV statistics assembled from galaxies with available $\mathrm{H} \alpha$ images.

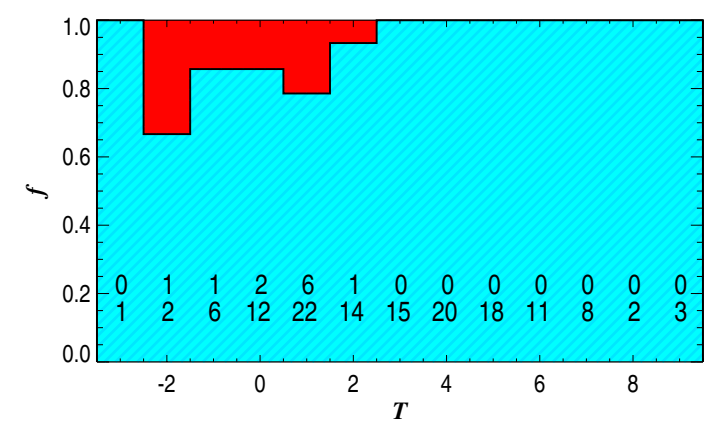

Fig. 3. Fraction of active and passive inner rings according to the FUV indicator as in Fig. 2, but now for inner rings in galaxies that have both FUV and $\mathrm{H} \alpha$ imaging available.

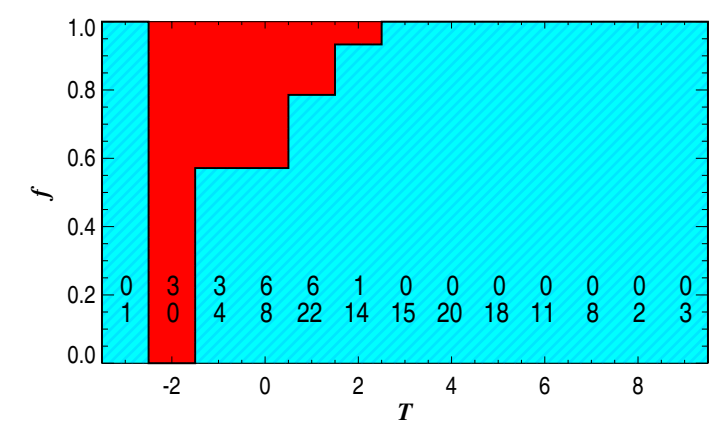

Fig. 4. As Fig. 2, but now using $\mathrm{H} \alpha$ emission as an indicator on whether inner rings are passive or active.

Because $\mathrm{H} \alpha$ imaging is only available for a part of the sample, I reproduced the plot for the FUV inner ring emission in Fig. 2 by using only FUV data that correspond to galaxies for which $\mathrm{H} \alpha$ is available (Fig. 3 and Table 1). This is used below to compare the fraction of passive inner rings according to the FUV and $\mathrm{H} \alpha$ indicators.

Figure 4 and Table 1 show the fraction of passive inner rings according to the $\mathrm{H} \alpha$ indicator. This fraction is equal to or larger than that of passive rings according to the FUV for all stages ( $28 \pm 5 \%$ of passive rings in the range $-3 \leq T \leq 2$ where all passive rings are found). This is because none of the rings lacking FUV emission have $\mathrm{H} \alpha$ emission, whereas the reverse is not always true.

While in the range of stages $-3 \leq T \leq 2$ the fraction of active inner features classified as ring-lenses is one in ten or less, 30-40\% of the passive inner features are ring-lenses (Table 2). Regarding inner features that are not classified as ringlenses, inner closed rings are equally frequent among the passive and the active rings ( $30-40 \%)$, but pseudorings are less frequent among passive features than among active ones $(\sim 30 \%$ vs. $\sim 60 \%$ ).
The passive or active status of a ring does not depend on the family (bar properties) of the host galaxy. Unbarred galaxies (SA) account for $\sim 30 \%$ of host galaxies for both passive and active rings in the stage range $-3 \leq T \leq 2$.

\section{Discussion}

As seen in Sect. 1, two mechanisms have been proposed for the formation of inner rings, namely the resonance and the manifold ones. I consider the resonance mechanism first.

If inner rings are the consequence of the star formation in gas gathered in orbits near resonances, one may expect that once the gas is exhausted, the ring will fade-out and disappear because of two factors. First, bright stars with a low mass-to-light ratio will die after several Myr. Second, radial migration will cause the ring to widen. Both effects would lower the surface brightness of the ring and will tend to make it indistinguishable from the stellar background of the galaxy. Of course, active rings may also have populations of old stars widened by radial migration, but they are likely to be outshone by the regions with recent star formation and thus would appear very sharp.

One piece of evidence that might indicate that rings become wider as they die is that as seen in Table 2, ring-lenses are roughly four times more frequent among passive features than among active features. It is unclear, however, whether the full width of ring-lenses can be explained by the radial migration of stars in passive rings. Alternatively, ring-lenses may form as a response of the old stellar population to the bar potential.

Under the assumption that the resonance scenario applies, an estimate of the dissolution time-scale of rings can be made from the data presented here and by knowing that $\mathrm{H} \alpha$ emission outlasts star formation by $20 \mathrm{Myr}$ and FUV emission outlasts star formation by $100 \mathrm{Myr}$ (Kennicutt 1998). The subsample of galaxies with both $\mathrm{H} \alpha$ and FUV imaging includes 19 rings without $\mathrm{H} \alpha$ emission. Eleven of those rings have no FUV emission. This means that eight rings stopped forming stars between 20 and $100 \mathrm{Myr}$ ago, and the remainder stopped forming stars longer than $100 \mathrm{Myr}$ ago. Assuming that the fraction of dissolving inner rings has been roughly constant for the past few hundreds of Myr, one can deduce that the ring dissolution time-scale is 200 Myr. This is a time of the order of an orbital period at the radius of inner rings.

However, this $\sim 200$ Myr estimated dissolution time-scale is a lower limit to the true dissolution time-scale. First, rings may form stars intermittently in recurrent episodes. It is therefore reasonable to assume that some of the passive rings may actually be reactivated at some point by some gas inflow. Such periodic activity has been reported in nuclear rings (Allard et al. 2006; Sarzi et al. 2007). Episodic star formation seems more likely in rings that have stopped forming stars more recently (those without $\mathrm{H} \alpha$ emission but with FUV emission), hence the dissolution time-scale underestimation. Second, $\mathrm{H} \alpha$ surveys may be biased against galaxies with little or no $\mathrm{H} \alpha$ emission. This would bias the surveys against galaxies with passive rings, and especially against those that cannot be reactivated, because if the galaxy still has some gas reserve that can be transferred to the ring, some residual star formation may remain elsewhere in the galaxy. As a consequence, the fraction of inner rings without either FUV or $\mathrm{H} \alpha$ emission might be underestimated.

In the framework of the manifold theory, passive rings are not necessarily dissolving. Indeed, manifolds can trap both stars and gas, and for galaxies with little or no gas, purely stellar rings are expected. However, it is still natural to expect passive rings to be wider. Indeed, stars can easily occupy the whole 
Table 2. Fraction of passive and active inner features with a given morphology as classified in Buta et al. (in prep.) for different star formation indicators in galaxies with $-3 \leq T \leq 2$.

\begin{tabular}{|c|c|c|c|c|c|c|}
\hline \multirow[t]{2}{*}{ Indicator } & \multicolumn{2}{|c|}{$\mathrm{rl}, \mathrm{rl}, \underline{\mathrm{r}} \mathrm{l}, \mathrm{r}^{\prime} \underline{1}, \mathrm{r}^{\prime} 1, \underline{\mathrm{r}}^{\prime} 1$} & \multicolumn{2}{|c|}{$r$} & \multicolumn{2}{|c|}{$\mathrm{rs}, \mathrm{rs}, \underline{\mathrm{rs}}$} \\
\hline & $P$ & $\overline{4}$ & $P$ & $A$ & $P^{-}$ & $-A$ \\
\hline FUV & $39 \pm 9 \%$ & $9 \pm 3 \%$ & $33 \pm 7 \%$ & $35 \pm 4 \%$ & $27 \pm 8 \%$ & $56 \pm 4 \%$ \\
\hline FUV $(\mathrm{H} \alpha)$ & $36 \pm 15 \%$ & $9 \pm 4 \%$ & $36 \pm 15 \%$ & $32 \pm 6 \%$ & $27 \pm 13 \%$ & $60 \pm 6 \%$ \\
\hline $\mathrm{H} \alpha$ & $32 \pm 11 \%$ & $6 \pm 3 \%$ & $32 \pm 11 \%$ & $33 \pm 7 \%$ & $37 \pm 11 \%$ & $61 \pm 7 \%$ \\
\hline
\end{tabular}

Notes. P and A stand for passive and active rings, respectively. FUV ( $\mathrm{H} \alpha)$ stands for the FUV statistics assembled from galaxies with available $\mathrm{H} \alpha$ images.

manifold phase space, but gas collisions would cause it (and also the younger generations of stars) to fill a smaller space and thus make the rings appear thinner (Athanassoula et al. 2009b). Whether this effect is enough to explain the full difference in width between regular rings and ring-lenses is not yet explored.

A large fraction of inner rings with a manifold origin could significantly change the dissolution time-scale estimated before. At the moment, no estimate is available on the fraction of inner rings caused by manifolds. Their existence is nearly certain, however, because the characteristic morphology of all types of outer rings, as well as the statistics of the shapes and sizes of both inner and outer rings in nearby galaxies, can be explained by the manifold theory (Athanassoula et al. 2009b, 2010). On the other hand, a set of $\sim 20 \mathrm{~N}$-body simulations with a fixed potential shows that at least in some cases only a minority of ring particles are trapped in manifolds (Rautiainen, priv. comm.). This, however, is not the case for the fully self-consistent simulations of Athanassoula (2012b). Additional study, both observational and numerical, is required to reveal whether manifolds can be easily populated and therefore are a widespread mechanism for shaping galaxy morphology.

In either the resonance or manifold frameworks the lack of passive rings in galaxies with $T \geq 3$ is naturally explained because in both cases, gas is available in these late-type galaxies to populate the orbits near resonances and/or the manifold orbits.

\section{Conclusions}

I used two indicators of recent star formation to check whether inner rings and pseudorings in a set of 319 nearby disc galaxies are passive (without signs of recent star formation) or active (with signs of recent star formation).

I showed that passive rings are only found in galaxies with stages $-3 \leq T \leq 2$. In that range of stages, $21 \pm 3 \%$ and $28 \pm 5 \%$ of rings are passive according to the FUV and $\mathrm{H} \alpha$ indicators, respectively. When a ring is passive in the FUV, it is also passive in $\mathrm{H} \alpha$, but the reverse is not always true. I found that $30-40 \%$ of passive inner rings are classified as ring-lenses in Buta et al. (in prep.). On the other hand, only $\sim 10 \%$ of active inner rings in the stage range $-3 \leq T \leq 2$ are ring-lenses. Although passive rings in both resonance and manifold theories are expected to be wider than their active counterparts, it is still unclear whether these two theories can account for the full transformation of regular inner rings into wide inner ring-lenses.

I estimate that if most inner rings have a resonance origin, a lower boundary for their dissolution time-scale is $200 \mathrm{Myr}$. This time-scale is of the order of one orbital period at the radius of inner rings.
Acknowledgements. The author thanks the referee, S. Ryder for useful comments. He thanks L. C. Ho, who gave the inspiration for this Letter and P. Rautiainen, H. Salo, J. H. Knapen and E. Athanassoula for useful discussions. This research is based in part on observations made with the Spitzer Space Telescope, which is operated by the Jet Propulsion Laboratory, California Institute of Technology under a contract with NASA. GALEX (Galaxy Evolution Explorer) is a NASA Small Explorer, launched in April 2003. We gratefully acknowledge NASA's support for construction, operation, and science analysis for the GALEX mission. This research has made use of the NASA/IPAC Extragalactic Database (NED) which is operated by the Jet Propulsion Laboratory, California Institute of Technology, under contract with the National Aeronautics and Space Administration. Based on observations made with the NASA/ESA Hubble Space Telescope, and obtained from the Hubble Legacy Archive, which is a collaboration between the Space Telescope Science Institute (STScI/NASA), the Space Telescope European Coordinating Facility (ST-ECF/ESA) and the Canadian Astronomy Data Centre (CADC/NRC/CSA).

\section{References}

Allard, E. L., Knapen, J. H., Peletier, R. F., \& Sarzi, M. 2006, MNRAS, 371, 1087

Athanassoula, E. 2012a, Proceedings of the XXIII Canary Islands Winter School of Astrophysics: Secular Evolution of Galaxies, eds. J. Falcon-Barroso, \& J. H. Knapen (Cambridge University Press) [arXiv: 1211.6752]

Athanassoula, E. 2012b, MNRAS, 426, L46

Athanassoula, E., Romero-Gómez, M., Bosma, A., \& Masdemont, J. J. 2009a, MNRAS, 400, 1706

Athanassoula, E., Romero-Gómez, M., \& Masdemont, J. J. 2009b, MNRAS, 394,67

Athanassoula, E., Romero-Gómez, M., Bosma, A., \& Masdemont, J. J. 2010, MNRAS, 407, 1433

Buta, R. 1991, ApJ, 370, 130

Comerón, S., Knapen, J. H., Beckman, J. E., et al. 2010, MNRAS, 402, 2462

Erwin, P., Vega Beltrán, J. C., \& Beckman, J. E. 2001, in The Central Kiloparsec of Starbursts and AGN: The La Palma Connection, eds. J. H. Knapen, J. E. Beckman, I. Shlosman, \& T. J. Mahoney, ASP Conf. Ser., 249, 171

Kennicutt, R. C. Jr. 1998, ARA\&A, 36, 189

Kim, W.-T., Seo, W.-Y., Stone, J. M., Yoon, D., \& Teuben, P. J. 2012, ApJ, 747, 60

Knapen, J. H., Whyte, L. F., de Blok, W. J. G., \& van der Hulst, J. M. 2004, A\&A, 423, 481

Knapen, J. H., Mazzuca, L. M., Böker, T., et al. 2006, A\&A, 448, 489

Laurikainen, E., Salo, H., Buta, R., \& Knapen, J. H. 2011, MNRAS, 418, 1452

Paturel, G., Petit, C., Prugniel, P., et al. 2003, A\&A, 412, 45

Rautiainen, P., \& Salo, H. 2000, A\&A, 362, 465

Romero-Gómez, M., Masdemont, J. J., Athanassoula, E., \& García-Gómez, C. 2006, A\&A, 453, 39

Romero-Gómez, M., Athanassoula, E., Masdemont, J. J., \& García-Gómez, C. 2007, A\&A, 472, 63

Sarzi, M., Allard, E. L., Knapen, J. H., \& Mazzuca, L. M. 2007, MNRAS, 380, 949

Schwarz, M. P. 1981, ApJ, 247, 77

Schwarz, M. P. 1984, MNRAS, 209, 93

Sheth, K., Regan, M., Hinz, J. L., et al. 2010, PASP, 122, 1397 
S. Comerón: Inner rings in disc galaxies: dead or alive

\section{Appendix A: Properties of the inner rings in the sample}

Table A.1. Properties of the inner rings in the sample.

\begin{tabular}{|c|c|c|c|c|c|c|}
\hline $\begin{array}{l}\text { ID } \\
\text { (1) }\end{array}$ & $\begin{array}{l}\text { Family } \\
\text { (2) }\end{array}$ & $\begin{array}{c}T \\
(3)\end{array}$ & $\begin{array}{l}\text { Kind } \\
\text { (4) }\end{array}$ & $\begin{array}{l}\text { FUV } \\
\text { (5) }\end{array}$ & $\begin{array}{l}\text { Survey } \\
\text { (6) }\end{array}$ & $\begin{array}{r}\mathrm{H} \alpha \\
(7)\end{array}$ \\
\hline ESO 013-016 & SB & 6 & rs & $\mathrm{A}$ & AIS & - \\
\hline ESO 202-041 & SB & 9 & rs & A & AIS & - \\
\hline ESO 404-012 & SAB & 3 & $\underline{\mathrm{r} s}$ & A & AIS & - \\
\hline ESO 407-014 & SA & 2 & $\mathrm{r}$ & A & MIS & - \\
\hline ESO 420-009 & SABB & 5 & rs & A & AIS & - \\
\hline ESO 422-005 & $\overline{\mathrm{SB}}$ & 8 & rs & A & AIS & - \\
\hline ESO 440-011 & SB & 6 & $\overline{\text { rs }}$ & A & NGS & - \\
\hline ESO 443-069 & SB & 8 & rs & A & AIS & - \\
\hline ESO 479-004 & SB & 7 & rs & A & GII & - \\
\hline ESO 482-035 & SB & 3 & rs & A & AIS & - \\
\hline ESO 508-007 & $\mathrm{SAB}$ & 7 & rs & A? & AIS & - \\
\hline ESO 510-059 & SB & 5 & rs & A & AIS & - \\
\hline ESO 532-022 & SB & 7 & $\overline{r s}$ & A & AIS & - \\
\hline ESO 547-005 & SAB & 9 & $\overline{\text { rs }}$ & A & MIS & - \\
\hline ESO 548-005 & SAB & 8 & rs & A & AIS & - \\
\hline ESO 572-018 & SAB & 3 & rs & A & AIS & - \\
\hline ESO 576-032 & SB & 5 & rs & A & AIS & - \\
\hline ESO 602-030 & SB & 7 & rs & A & AIS & - \\
\hline IC 0749 & SB & 6 & rs & A & AIS & A \\
\hline IC 1014 & SB & 9 & $\mathrm{r}$ & A & AIS & - \\
\hline IC 1067 & SB & 3 & $\mathrm{r}$ & A & GII & - \\
\hline IC 1265 & SA & 0 & $\mathrm{r}$ & $\mathrm{A}$ ? & AIS & - \\
\hline IC 1438 & SAB & 0 & $r^{\prime} 1$ & A & AIS & - \\
\hline IC 1954 & SB & 6 & rs & A & GII & - \\
\hline IC 2969 & SA & 7 & $\overline{\mathrm{r}}$ & A & AIS & - \\
\hline IC 3102 & SAB & 0 & $\underline{\mathrm{rs}}$ & A & GII & - \\
\hline IC 3267 & SA & 1 & rs & A & GII & - \\
\hline IC 4214 & SAB & 0 & $r^{\prime} 1$ & A & AIS & - \\
\hline IC 4237 & $\overline{\mathrm{SB}}$ & 3 & $\mathrm{r}$ & A & AIS & - \\
\hline IC 5240 & SB & 0 & $\mathrm{r}$ & A & AIS & A \\
\hline IC 5267 & SA & 0 & $\mathrm{r}$ & A & AIS & - \\
\hline NGC 0150 & SAB & 2 & rs & A & AIS & - \\
\hline NGC 0210 & $\mathrm{SAB}$ & 2 & $\mathrm{r}^{\bar{\prime} 1}$ & A & GII & A \\
\hline NGC 0255 & SB & 6 & rs & A & AIS & - \\
\hline NGC 0289 & $\mathrm{SA} \underline{\mathrm{B}}$ & 2 & rs, $\underline{\text { rs }}$ & $\mathrm{A}, \mathrm{A}$ & MIS & - \\
\hline NGC 0470 & $S \underline{A} \bar{B}$ & 2 & rs & A & MIS & A \\
\hline NGC 0473 & $\overline{\mathrm{SA}}$ & -1 & $\overline{\mathrm{r}}$ & A & AIS & A \\
\hline NGC 0488 & SA & 1 & $\mathrm{rl}$ & $\mathrm{P}$ & MIS & $\mathrm{P}$ \\
\hline NGC 0600 & SB & 7 & rs & A & AIS & - \\
\hline NGC 0613 & SB & 3 & $\underline{\mathrm{rs}}$ & A & AIS & A \\
\hline NGC 0658 & SA & 4 & rs & A & MIS & A \\
\hline NGC 0691 & SA & 2 & $\mathrm{rs}, \mathrm{r}$ & $\mathrm{A}, \mathrm{A}$ & AIS & $\mathrm{A}, \mathrm{A}$ \\
\hline NGC 0701 & SB & 7 & $\underline{\mathrm{rs}}$ & A & MIS & - \\
\hline NGC 0718 & SAB & 1 & rs & P? & AIS & $\mathrm{P}$ \\
\hline NGC 0864 & SAB & 4 & rs & A & MIS & A \\
\hline NGC 0908 & $\mathrm{SA}^{-}$ & 3 & $\overline{\mathrm{rs}}$ & A & GII & - \\
\hline NGC 0936 & SB & -1 & $\underline{\mathrm{rs}}$ & $\mathrm{P}$ & GII & - \\
\hline NGC 0941 & $\mathrm{SAB}$ & 5 & $\overline{\mathrm{r}}$ & A & GII & A \\
\hline NGC 0986 & $\overline{\mathrm{SB}}$ & 2 & rs & A & NGS & A \\
\hline NGC 1022 & SAB & 0 & $\underline{\mathrm{r} s}$ & A & NGS & $\mathrm{P}$ \\
\hline NGC 1073 & SB & 5 & $\overline{\mathrm{rs}}$ & A & GII & A \\
\hline NGC 1079 & $\mathrm{SA} B$ & -1 & $\underline{\mathrm{r} s}$ & A & MIS & - \\
\hline NGC 1087 & $\overline{\mathrm{SB}}$ & 7 & $\overline{\mathrm{r}} \underline{\mathrm{s}}$ & A & MIS & A \\
\hline NGC 1097 & SB & 3 & $\overline{\mathrm{rs}}$ & A & NGS & A \\
\hline NGC 1179 & $\mathrm{SAB}$ & 6 & rs & A & MIS & A \\
\hline NGC 1187 & $\mathrm{SA} \underline{\bar{B}}$ & 4 & $\overline{\mathrm{rs}}$ & A & GII & A \\
\hline NGC 1201 & $\mathrm{SA} \overline{\mathrm{B}}$ & -2 & $\mathrm{r}^{\prime} 1$ & P? & AIS & - \\
\hline NGC 1232 & SAB & 5 & rs & A & AIS & - \\
\hline
\end{tabular}

Notes. ID (Col. 1) refers to the galaxy name, family (Col. 2) to its bar classification and and $T$ (Col. 3) to its stage from Buta et al. (in prep.) and NIRSOS. Kind (Col. 4) indicates the ring classification by Buta et al. (in prep.) and NIRSOS. FUV (Col. 5) indicates whether a given ring emits in the ultraviolet continuum ("A") or not ("P"). The Survey column (Col. 6) indicates to which GALEX survey the FUV images used here belong. H $\alpha$ (Col. 7) indicates whether a given ring is seen in continuum-subtracted $\mathrm{H} \alpha$ images ("A"), or not ("P"). Uncertain detection statuses are indicated by "?" in Cols. 5 and 7. 
Table A.1. continued.

\begin{tabular}{|c|c|c|c|c|c|c|}
\hline $\begin{array}{l}\text { ID } \\
\text { (1) }\end{array}$ & $\begin{array}{c}\text { Family } \\
\text { (2) }\end{array}$ & $\begin{array}{c}T \\
(3)\end{array}$ & $\begin{array}{l}\text { Kind } \\
\text { (4) }\end{array}$ & $\begin{array}{c}\text { FUV } \\
(5)\end{array}$ & $\begin{array}{c}\text { Survey } \\
\text { (6) }\end{array}$ & $\begin{array}{l}\mathrm{H} \alpha \\
(7)\end{array}$ \\
\hline NGC 1297 & SA & -2 & $\mathrm{rl}$ & P? & MIS & - \\
\hline NGC 1310 & SB & 6 & rs & A & NGS & - \\
\hline NGC 1317 & SAB & 0 & $r^{\prime} 1$ & A & NGS & $\mathrm{P}$ \\
\hline NGC 1326 & SAB & -1 & $\mathrm{r}$ & A & NGS & $\mathrm{P}$ \\
\hline NGC 1350 & SAB & 0 & $\mathrm{r}$ & A & NGS & A \\
\hline NGC 1357 & SA & 0 & $\underline{\mathrm{rs}}$ & A & AIS & - \\
\hline NGC 1365 & SB & 4 & rs & A & NGS & - \\
\hline NGC 1367 & SAB & 0 & $\overline{\mathrm{rs}}$ & A & GII & $\mathrm{P}$ \\
\hline NGC 1385 & SB & 8 & rs & A & NGS & - \\
\hline NGC 1398 & SB & 1 & rs & A & GII & A \\
\hline NGC 1433 & SB & 1 & $\bar{r}$ & A & AIS & A \\
\hline NGC 1440 & SB & -2 & $\underline{\mathrm{rs}}$ & P? & AIS & - \\
\hline NGC 1452 & SB & 0 & $\mathrm{r}$ & A? & AIS & - \\
\hline NGC 1493 & SB & 5 & rs & A & GII & A \\
\hline NGC 1512 & SB & 1 & $\mathrm{r}$ & A & NGS & A \\
\hline NGC 1553 & SA & -1 & $\mathrm{rl}$ & $\mathrm{P}$ & NGS & - \\
\hline NGC 1566 & SAB & 3 & $r^{\prime} 1$ & A & NGS & A \\
\hline NGC 1640 & SB & 1 & $\mathrm{r}$ & A & AIS & - \\
\hline NGC 1672 & $\mathrm{SAB}$ & 3 & rs & A & NGS & A \\
\hline NGC 2460 & $\mathrm{~S} \underline{\mathrm{A}} \overline{\mathrm{B}}$ & 1 & rs & A & AIS & A \\
\hline NGC 2523 & SB & 2 & $\mathrm{r}$ & A & AIS & - \\
\hline NGC 2604 & SB & 5 & rs & A & MIS & A \\
\hline NGC 2608 & SAB & 3 & $\overline{\mathrm{rs}}$ & A & MIS & A \\
\hline NGC 2633 & $\mathrm{SA} \overline{\mathrm{B}}$ & 3 & $\overline{\mathrm{rs}}$ & A & AIS & A \\
\hline NGC 2681 & SAB & 0 & rs & A & NGS & $\mathrm{P}$ ? \\
\hline NGC 2775 & $\overline{\mathrm{SA}}$ & -1 & $\underline{\mathrm{r}} \mathrm{s}$ & A & MIS & A \\
\hline NGC 2780 & SB & 1 & rs & A & AIS & - \\
\hline NGC 2782 & SA & 1 & rs & A & NGS & - \\
\hline NGC 2787 & SB & -2 & $\mathrm{r}$ & A & AIS & $\mathrm{P}$ \\
\hline NGC 2805 & SÁB & 5 & rs & A & AIS & A \\
\hline NGC 2859 & $\mathrm{~S} \overline{\mathrm{A}} \mathrm{B}$ & -1 & $\mathrm{r} \bar{l}$ & A? & GII & $\mathrm{P}$ \\
\hline NGC 2906 & SA & 3 & rs & A & MIS & - \\
\hline NGC 2950 & SAB & -1 & $\mathrm{r} \underline{\mathrm{l}}$ & $\mathrm{P}$ & AIS & $\mathrm{P}$ \\
\hline NGC 2962 & $\mathrm{~S} \underline{\mathrm{A}} \overline{\mathrm{B}}$ & -1 & $\mathrm{rl}$ & $\mathrm{P}$ & MIS & - \\
\hline NGC 2964 & SA-B & 3 & rs & A & NGS & - \\
\hline NGC 2966 & SAB & 1 & $r^{\prime} 1$ & A & MIS & - \\
\hline NGC 2967 & SAB & 5 & rs & A & MIS & - \\
\hline NGC 2968 & SB & -1 & rs & $\mathrm{P}$ & NGS & - \\
\hline NGC 2974 & SA & 0 & $\overline{\mathrm{r}}$ & A & GII & - \\
\hline NGC 3031 & SA & 1 & $\mathrm{rs}, \mathrm{r}$ & $\mathrm{A}, \mathrm{P}$ & GII & A, P \\
\hline NGC 3032 & SA & -2 & $\mathrm{rs}, \mathrm{r}$ & $\mathrm{P}$ & GII & - \\
\hline NGC 3061 & SAB & 3 & $\underline{\text { rs }}$ & A & AIS & - \\
\hline NGC 3147 & SABB & 3 & rs & A & NGS & - \\
\hline NGC 3166 & $\overline{\mathrm{SB}}$ & -1 & $\mathrm{rl}$ & A & GII & - \\
\hline NGC 3184 & SA & 4 & rs & A & AIS & A \\
\hline NGC 3185 & SAB & 1 & $\underline{\mathrm{rs}}$ & A & NGS & A \\
\hline NGC 3245 & SAB & -2 & rs & A? & GII & $\mathrm{P}$ \\
\hline NGC 3344 & SAB & 4 & $\mathrm{r}$ & A & NGS & A \\
\hline NGC 3346 & SB & 6 & rs & A & AIS & - \\
\hline NGC 3351 & SB & 1 & $\mathrm{r}$ & A & NGS & A \\
\hline NGC 3359 & SB & 7 & rs & A & NGS & A \\
\hline NGC 3368 & SAB & -1 & $\underline{\mathrm{rs}}$ & A & NGS & A \\
\hline NGC 3380 & $\mathrm{SA} \underline{\mathrm{B}}$ & 0 & $\underline{\mathrm{r}} \mathrm{s}$ & A & AIS & - \\
\hline NGC 3381 & SB & 8 & $\mathrm{r} \underline{s}$ & A & AIS & - \\
\hline NGC 3455 & SA & 5 & $\overline{\mathrm{rs}}$ & A & GII & - \\
\hline NGC 3485 & $\mathrm{SAB}$ & 3 & $\underline{\text { rs }}$ & A & GII & A \\
\hline NGC 3486 & SAB & 5 & $\mathrm{r}$ & A & GII & A \\
\hline NGC 3504 & $\mathrm{SAB}$ & 1 & $\underline{\mathrm{rs}}$ & A & AIS & A \\
\hline NGC 3513 & SB & 5 & $\overline{\mathrm{r}} \underline{\mathrm{s}}$ & A & GII & A \\
\hline NGC 3547 & SB & 6 & rs & A & GII & - \\
\hline NGC 3583 & SAB & 3 & rs & A & AIS & - \\
\hline NGC 3611 & SA & 1 & $\mathrm{r}$ & A & MIS & - \\
\hline NGC 3614 & SA & 4 & $\mathrm{r}$ & A & AIS & - \\
\hline NGC 3637 & SB & -1 & $\mathrm{rl}$ & P? & AIS & - \\
\hline NGC 3642 & SA & 2 & $\mathrm{rl}$ & $\mathrm{P}$ ? & GII & $\mathrm{P}$ ? \\
\hline NGC 3664 & SB & 9 & rs & A & MIS & - \\
\hline
\end{tabular}


S. Comerón: Inner rings in disc galaxies: dead or alive

Table A.1. continued.

\begin{tabular}{|c|c|c|c|c|c|c|}
\hline $\begin{array}{l}\text { ID } \\
\text { (1) }\end{array}$ & $\begin{array}{c}\text { Family } \\
\text { (2) }\end{array}$ & $\begin{array}{c}T \\
\text { (3) }\end{array}$ & $\begin{array}{c}\text { Kind } \\
\text { (4) }\end{array}$ & $\begin{array}{c}\text { FUV } \\
\text { (5) }\end{array}$ & $\begin{array}{c}\text { Survey } \\
\text { (6) }\end{array}$ & $\begin{array}{l}\mathrm{Ho} \\
(7)\end{array}$ \\
\hline NGC 3673 & SAB & 1 & rs & $\bar{A}$ & AIS & - \\
\hline NGC 3681 & $\mathrm{SA} \overline{\mathrm{B}}$ & 1 & rs & A & AIS & - \\
\hline NGC 3683A & SAB & 4 & rs & A & AIS & - \\
\hline NGC 3687 & SAB & 2 & rs & A & AIS & - \\
\hline NGC 3691 & $\mathrm{SB}$ & 9 & $\mathrm{r}$ & A & AIS & - \\
\hline NGC 3705 & SAB & 3 & rs & A & AIS & A \\
\hline NGC 3726 & SAB & 4 & $\bar{r}$ & A & AIS & A \\
\hline NGC 3729 & $\mathrm{SB}$ & 0 & $\mathrm{r}$ & A & GII & A \\
\hline NGC 3780 & SA & 4 & rs & A & AIS & - \\
\hline NGC 3782 & SB & 8 & rs & A & AIS & A \\
\hline NGC 3786 & SA & 0 & $\mathrm{r}$ & A & AIS & - \\
\hline NGC 3870 & SB & -2 & rs & $\mathrm{A}$ ? & GII & - \\
\hline NGC 3887 & $\mathrm{SAB}$ & 4 & rs & A & GII & A \\
\hline NGC 3888 & $\mathrm{SA}$ & 3 & rs & A & AIS & - \\
\hline NGC 3892 & SAB & -1 & $r^{\prime} 1$ & $\mathrm{P}$ & AIS & - \\
\hline NGC 3900 & $\mathrm{SA}^{-}$ & 0 & $\mathrm{r}$ & A & AIS & - \\
\hline NGC 3945 & SB & -1 & $\mathrm{rl}$ & A & AIS & - \\
\hline NGC 3949 & SAB & 5 & rs & A & AIS & A \\
\hline NGC 4030 & SA & 4 & rs & A & MIS & A \\
\hline NGC 4037 & SAB & 5 & rs & A & AIS & A \\
\hline NGC 4041 & SAB & 5 & rs & A & GII & A \\
\hline NGC 4045 & $S \bar{A} B$ & 2 & rs & A & GII & A \\
\hline NGC 4050 & SAB & 1 & rs & A & AIS & - \\
\hline NGC 4051 & $\mathrm{SA} \overline{\mathrm{B}}$ & 3 & rs & A & AIS & A \\
\hline NGC 4067 & SB & 2 & rs & A & GII & - \\
\hline NGC 4116 & SB & 7 & rs & A & MIS & A \\
\hline NGC 4123 & SB & 3 & rs & A & AIS & A \\
\hline NGC 4136 & $\mathrm{SAB}$ & 4 & rs & A & GII & A \\
\hline NGC 4138 & $\overline{\mathrm{SA}}$ & -1 & $\mathrm{r}$ & A & NGS & - \\
\hline NGC 4141 & SB & 7 & rs & A & AIS & - \\
\hline NGC 4145 & SAB & 7 & $\overline{\mathrm{rs}}$ & A & AIS & A \\
\hline NGC 4162 & SA & 5 & $\mathrm{r}$ & A? & AIS & - \\
\hline NGC 4189 & SAB & 4 & rs & A & GII & A \\
\hline NGC 4212 & $\mathrm{SA}$ & 3 & $\overline{\mathrm{rs}}$ & A & GII & A \\
\hline NGC 4234 & SB & 9 & rs & A & AIS & A \\
\hline NGC 4245 & SB & -1 & $\mathrm{r}$ & A & GII & A \\
\hline NGC 4250 & SAB & -1 & $\mathrm{rl}$ & A & AIS & - \\
\hline NGC 4298 & SA & 4 & rs & A & GII & A \\
\hline NGC 4303 & SAB & 5 & rs & A & NGS & A \\
\hline NGC 4309 & SAB & -2 & $\mathrm{rl}$ & A & GII & - \\
\hline NGC 4314 & SB & 1 & $\mathrm{rl}$ & $\mathrm{P}$ & NGS & $\mathrm{P}$ \\
\hline NGC 4321 & SAB & 4 & rs & A & GII & A \\
\hline NGC 4336 & SAB & 0 & $\overline{\mathrm{r}}$ & $\mathrm{P}$ & AIS & - \\
\hline NGC 4339 & SA & -2 & $\mathrm{r}$ & A? & AIS & - \\
\hline NGC 4340 & SB & -1 & $r$ & $\mathrm{P}$ ? & AIS & - \\
\hline NGC 4371 & SB & -2 & $\mathrm{r}$ & $\mathrm{P}$ & DIS & - \\
\hline NGC 4380 & SA & 2 & $\mathrm{r}$ & A & GII & A \\
\hline NGC 4385 & SAB & 2 & rs & A & AIS & A \\
\hline NGC 4394 & $\mathrm{SB}$ & 0 & rs & A & AIS & A \\
\hline NGC 4405 & SAB & 1 & rs & A & NGS & A \\
\hline NGC 4411A & $\mathrm{SB}$ & 6 & $\overline{\mathrm{rs}}$ & A & GII & - \\
\hline NGC 4412 & SAB & 4 & rs & A & AIS & A \\
\hline NGC 4413 & $\mathrm{SB}$ & 2 & rs & A & NGS & A \\
\hline NGC 4414 & SA & 4 & $\mathrm{rl}$ & A & NGS & A \\
\hline NGC 4416 & SB & 8 & rs & A & GII & A \\
\hline NGC 4430 & SAB & 8 & $\underline{\mathrm{rs}}$ & A & AIS & - \\
\hline NGC 4450 & SAB & 1 & rs & A & AIS & A \\
\hline NGC 4454 & SAB & 0 & $\mathrm{r}$ & A & AIS & - \\
\hline NGC 4477 & SB & 1 & $\mathrm{r}$ & $\mathrm{P}$ ? & GII & - \\
\hline NGC 4491 & SB & 0 & rs & $\mathrm{P}$ ? & NGS & - \\
\hline NGC 4492 & SA & -3 & $\underline{\mathrm{rs}}$ & A & AIS & A \\
\hline NGC 4496A & SB & 7 & $\overline{\mathrm{rs}}$ & A & AIS & - \\
\hline NGC 4498 & SB & 7 & rs & A & GII & A \\
\hline NGC 4501 & SA & 3 & rs & A & AIS & A \\
\hline NGC 4504 & SAB & 5 & rs & A & GII & - \\
\hline NGC 4519 & SAB & 6 & rs & A & GII & A \\
\hline
\end{tabular}


Table A.1. continued.

\begin{tabular}{|c|c|c|c|c|c|c|}
\hline $\begin{array}{l}\text { ID } \\
\text { (1) }\end{array}$ & $\begin{array}{c}\text { Family } \\
\text { (2) }\end{array}$ & $\begin{array}{c}T \\
(3)\end{array}$ & $\begin{array}{c}\text { Kind } \\
\text { (4) }\end{array}$ & $\begin{array}{c}\text { FUV } \\
(5)\end{array}$ & $\begin{array}{c}\text { Survey } \\
\text { (6) }\end{array}$ & $\begin{array}{l}\mathrm{H} \alpha \\
\text { (7) }\end{array}$ \\
\hline NGC 4528 & SB & -2 & $r$ & P? & NGS & - \\
\hline NGC 4531 & SA & 1 & rs & A & NGS & A \\
\hline NGC 4540 & SAB & 9 & $\overline{\mathrm{rs}}$ & A & AIS & A \\
\hline NGC 4548 & SB & 1 & rs & A & GII & A \\
\hline NGC 4567 & SA & 4 & rs & A & GII & A \\
\hline NGC 4579 & SB & 1 & rs & A & NGS & A \\
\hline NGC 4580 & SA & 1 & $\underline{\mathrm{rs}}, \mathrm{rs}$ & $\mathrm{P}, \mathrm{A}$ & GII & $\mathrm{P}, \mathrm{A}$ \\
\hline NGC 4593 & SB & 1 & rs & A & AIS & - \\
\hline NGC 4596 & SB & 0 & rs & $\mathrm{P}$ & GII & - \\
\hline NGC 4618 & SB & 9 & rs & A & NGS & A \\
\hline NGC 4639 & SB & 2 & $\underline{\mathrm{rs}}$ & A & AIS & A \\
\hline NGC 4643 & SB & -2 & $\mathrm{r}$ & P? & AIS & $\mathrm{P}$ \\
\hline NGC 4651 & SA & 4 & $\underline{\text { rs }}$ & A & GII & A \\
\hline NGC 4654 & SB & 6 & $\mathrm{rs}$ & A & AIS & A \\
\hline NGC 4680 & SAB & 3 & rs & A & AIS & - \\
\hline NGC 4698 & SA & 0 & $\mathrm{r}, \mathrm{r}$ & $\mathrm{A}, \mathrm{P}$ & GII & A, P \\
\hline NGC 4713 & SAB & 5 & rs & A & AIS & A \\
\hline NGC 4725 & $\mathrm{SA} \overline{\mathrm{B}}$ & 1 & $\mathrm{r}$ & A & AIS & A \\
\hline NGC 4736 & SABB & 1 & $\mathrm{rl}$ & A & NGS & A \\
\hline NGC 4750 & $\overline{\mathrm{SA}}$ & 1 & $\underline{\mathrm{rs}}$ & A & AIS & A \\
\hline NGC 4772 & SA & 0 & $\mathrm{r}$ & A & MIS & A \\
\hline NGC 4779 & SB & 3 & rs & A & AIS & - \\
\hline NGC 4793 & SA & 5 & rs & A & GII & A \\
\hline NGC 4800 & SA & 1 & rs & A & AIS & A \\
\hline NGC 4814 & SA & 4 & $\mathrm{r} \underline{\mathrm{s}}$ & A & GII & - \\
\hline NGC 4826 & SA & 1 & $\mathrm{rs}, \mathrm{r}$ & $\mathrm{P}, \mathrm{A}$ & CAI & $\mathrm{P}, \mathrm{A}$ \\
\hline NGC 4880 & SA-B & -1 & $\mathrm{rl}$ & $\mathrm{P}$ & AIS & - \\
\hline NGC 4897 & $\mathrm{SAB}$ & 3 & $\underline{\mathrm{rs}}$ & A & GII & - \\
\hline NGC 4902 & $\mathrm{SB}^{-}$ & 3 & $\underline{\text { rss }}$ & A & AIS & - \\
\hline NGC 4941 & SA & 0 & $\overline{\mathrm{r} s}$ & A & AIS & - \\
\hline NGC 4961 & SB & 4 & $\overline{\text { rs }}$ & A & GII & - \\
\hline NGC 4995 & SAB & 2 & rs & A & AIS & - \\
\hline NGC 5033 & SA & 5 & rs & A & AIS & A \\
\hline NGC 5055 & SA & 4 & $\mathrm{rs}, \mathrm{rl}$ & A, A & NGS & $\mathrm{A}, \mathrm{A}$ \\
\hline NGC 5068 & SB & 7 & rs & A & GII & A \\
\hline NGC 5101 & SB & 0 & $\underline{\text { rs }}$ & A & AIS & - \\
\hline NGC 5105 & SAB & 6 & $\overline{\mathrm{r}} \underline{\mathrm{s}}$ & A & AIS & - \\
\hline NGC 5112 & SB & 6 & $\overline{\mathrm{rs}}$ & A & AIS & A \\
\hline NGC 5134 & $\mathrm{SAB}$ & 0 & $\overline{\mathrm{rs}}$ & A & AIS & - \\
\hline NGC 5145 & SA & -1 & $\mathrm{r}$ & A & AIS & - \\
\hline NGC 5194 & SABB & 4 & rs & A & GII & A \\
\hline NGC 5195 & $\mathrm{SA} B$ & 0 & $\mathrm{r}$ & P? & GII & P? \\
\hline NGC 5205 & SB & 2 & rs & A & AIS & - \\
\hline NGC 5218 & SB & 1 & rs & A & AIS & - \\
\hline NGC 5300 & $\mathrm{~S} \underline{\mathrm{AB}}$ & 5 & rs & A & AIS & - \\
\hline NGC 5313 & $\overline{\mathrm{SA}}$ & 3 & $\overline{\mathrm{r}}$ & A & AIS & - \\
\hline NGC 5334 & SB & 6 & rs & A & AIS & A \\
\hline NGC 5339 & SB & 2 & rs & A & AIS & - \\
\hline NGC 5347 & SB & 1 & $\underline{\mathrm{rs}}$ & A & AIS & A \\
\hline NGC 5350 & SB & 3 & $\underline{\text { rs }}$ & A & AIS & - \\
\hline NGC 5364 & SA & 3 & $\bar{r}$ & A & DIS & A \\
\hline NGC 5371 & SAB & 3 & rs & A & AIS & A \\
\hline NGC 5375 & SB & 1 & $\underline{\mathrm{rs}}$ & A & GII & - \\
\hline NGC 5376 & SA & 2 & $\underline{\mathrm{r} s}$ & A & AIS & - \\
\hline NGC 5383 & SB & 1 & $\overline{\mathrm{rs}}$ & A & AIS & - \\
\hline NGC 5426 & SAB & 5 & rs & A & GII & - \\
\hline NGC 5457 & SAB & 5 & rs & A & GII & A \\
\hline NGC 5534 & $\overline{\mathrm{SB}}$ & 1 & $\overline{\mathrm{rs}}$ & A & AIS & A \\
\hline NGC 5595 & SAB & 6 & $\overline{\mathrm{rs}}$ & A & AIS & - \\
\hline NGC 5600 & SB & 8 & rs & A & AIS & - \\
\hline NGC 5636 & SAB & 0 & $\mathrm{r}$ & A & MIS & - \\
\hline NGC 5665 & SAB & 5 & rs & A & AIS & - \\
\hline NGC 5668 & SAB & 6 & rs & A & CAI & A \\
\hline NGC 5669 & SB & 7 & rs & A & AIS & A \\
\hline NGC 5678 & SA & 3 & $\underline{\mathrm{rs}}$ & A & AIS & - \\
\hline NGC 5701 & SB & 0 & $\mathrm{rl}$ & $\mathrm{P}$ & MIS & - \\
\hline
\end{tabular}


S. Comerón: Inner rings in disc galaxies: dead or alive

Table A.1. continued.

\begin{tabular}{|c|c|c|c|c|c|c|}
\hline $\begin{array}{l}\text { ID } \\
\text { (1) }\end{array}$ & $\begin{array}{c}\text { Family } \\
\text { (2) }\end{array}$ & $\begin{array}{c}T \\
(3)\end{array}$ & $\begin{array}{l}\text { Kind } \\
\text { (4) }\end{array}$ & $\begin{array}{c}\text { FUV } \\
(5)\end{array}$ & $\begin{array}{c}\text { Survey } \\
\text { (6) }\end{array}$ & $\begin{array}{l}\mathrm{Ho} \\
\text { (7) }\end{array}$ \\
\hline NGC 5713 & SB & 2 & rs & $\mathrm{A}$ & MIS & $\mathrm{A}$ \\
\hline NGC 5728 & SB & 0 & rs & A & AIS & A \\
\hline NGC 5740 & SAB & 2 & $\bar{r}$ & A & MIS & - \\
\hline NGC 5757 & $\mathrm{SB}^{-}$ & 2 & rs & A & AIS & A \\
\hline NGC 5768 & SAB & 4 & rs & A & MIS & - \\
\hline NGC 5770 & SAB & -1 & $\mathrm{r}$ & $\mathrm{P}$ & GII & - \\
\hline NGC 5806 & SAB & 2 & rs & A & GII & A \\
\hline NGC 5821 & SAB & 1 & $\mathrm{r}$ & A & AIS & - \\
\hline NGC 5850 & $\overline{\mathrm{SB}}$ & 2 & $\mathrm{r}$ & A & MIS & A \\
\hline NGC 5892 & SB & 6 & rs & A & AIS & - \\
\hline NGC 5915 & SA & 5 & $\bar{r}$ & A & GII & A \\
\hline NGC 5921 & SB & 3 & rs & A & AIS & A \\
\hline NGC 5930 & SAB & 0 & rs & A & AIS & - \\
\hline NGC 5957 & SB & 1 & $\underline{\mathrm{rs}}$ & A & GII & - \\
\hline NGC 5962 & SAB & 5 & rs & A & NGS & A \\
\hline NGC 5964 & SB & 6 & rs & A & GII & A \\
\hline NGC 6012 & SB & 2 & $\bar{r}$ & A & GII & - \\
\hline NGC 6014 & SAB & 0 & rs & A? & AIS & - \\
\hline NGC 6267 & $\mathrm{SB}$ & 3 & rs & A & AIS & - \\
\hline NGC 6278 & SA & -2 & $\mathrm{r}$ & P? & AIS & - \\
\hline NGC 6412 & SB & 6 & rs & A & AIS & A \\
\hline NGC 6902 & SABB & 1 & $\underline{\mathrm{rs}}$ & A & NGS & - \\
\hline NGC 7070 & $\overrightarrow{\mathrm{SB}}$ & 5 & $\mathrm{r} \underline{\mathrm{s}}$ & A & AIS & - \\
\hline NGC 7098 & SAB & 0 & $r^{\prime} 1$ & A & AIS & A \\
\hline NGC 7107 & SB & 8 & rs & A & AIS & - \\
\hline NGC 7179 & SB & 0 & $\mathrm{r}$ & A & GII & - \\
\hline NGC 7205 & SA & 4 & rs & A & AIS & A \\
\hline NGC 7290 & SA & 3 & rs & A & AIS & - \\
\hline NGC 7378 & SA & 0 & $\underline{\mathrm{rs}}$ & A & AIS & - \\
\hline NGC 7418 & SAB & 5 & rs & A & NGS & - \\
\hline NGC 7421 & SB & 2 & rs & A & NGS & - \\
\hline NGC 7424 & SB & 6 & rs & A & GII & A \\
\hline NGC 7496 & SB & 3 & $\mathrm{rs}$ & A & NGS & - \\
\hline NGC 7513 & SB & 1 & rs & A & AIS & - \\
\hline NGC 7531 & SAB & 1 & $\mathrm{r}$ & A & GII & - \\
\hline NGC 7552 & SB & 1 & rs & A & NGS & A \\
\hline NGC 7716 & $\mathrm{~S} \underline{\mathrm{AB}}$ & 2 & $\mathrm{r}$ & A & MIS & - \\
\hline NGC 7723 & $\overline{\mathrm{SB}}$ & 2 & rs & A & MIS & - \\
\hline NGC 7742 & SA & -1 & $\mathrm{r}, \mathrm{r}$ & $\mathrm{A}, \mathrm{A}$ & GII & - \\
\hline NGC 7743 & SAB & 1 & rs & $\mathrm{P}$ & AIS & - \\
\hline NGC 7755 & SAB & 4 & rs & A & AIS & - \\
\hline PGC 003853 & $\mathrm{SB}$ & 6 & $\underline{\mathrm{rs}}$ & A & AIS & - \\
\hline PGC 006626 & SB & 6 & rs & A & AIS & - \\
\hline PGC 012633 & SAB & 2 & rs & A & MIS & - \\
\hline PGC 012664 & $\mathrm{SAB}$ & 6 & rs & A & MIS & - \\
\hline PGC 032091 & $\mathrm{SA} \overline{\mathrm{B}}$ & 5 & $\overline{\mathrm{rs}}$ & A & AIS & - \\
\hline PGC 038250 & $\mathrm{SAB}$ & 9 & rs & A & AIS & - \\
\hline PGC 044735 & SAB & 8 & rs & A & GII & - \\
\hline PGC 044952 & SA & 7 & $\mathrm{r}$ & A & AIS & - \\
\hline PGC 047721 & SA & 2 & $\underline{\mathrm{rs}}, \mathrm{r}, \mathrm{r}$ & $\mathrm{A}, \mathrm{A} ?, \mathrm{~A} ?$ & AIS & - \\
\hline PGC 048179 & SB & 6 & rs & A & GII & A \\
\hline PGC 054944 & SB & 7 & rs & A & AIS & - \\
\hline UGC 01551 & SB & 6 & $\overline{\mathrm{rs}}$ & A & GII & - \\
\hline UGC 04867 & SB & 7 & $\mathrm{rs}$ & A & AIS & - \\
\hline UGC 06023 & SAB & 5 & $\underline{\mathrm{rs}}$ & A & AIS & A \\
\hline UGC 06309 & SB & 5 & $\mathrm{r} \underline{\mathrm{s}}$ & A & AIS & - \\
\hline UGC 07184 & SB & 7 & rs & A & MIS & - \\
\hline UGC 08155 & SA & 1 & rs & A & AIS & - \\
\hline UGC 09356 & SAB & 4 & rs & A & AIS & - \\
\hline UGC 09569 & SB & 5 & rs & A & AIS & - \\
\hline UGC 10054 & SB & 7 & $\mathrm{rs}$ & A & AIS & A \\
\hline UGC 10791 & SB & 7 & rs & A & NGS & - \\
\hline UGC 12151 & SB & 7 & rs & A & MIS & - \\
\hline
\end{tabular}

